\title{
A New Multichannel Fourier Transform Spectrometer
}

\author{
Tyler E. Nordgren \\ U.S. Naval Observatory, NPOI, PO Box 447, Rural Rte 14, Flagstaff, \\ $A Z 86001$
}

Arsen R. Hajian

U.S. Naval Observatory, Department of Astrometry, 3450

Massachusetts Ave., NW, Washington DC 20392-5420

\begin{abstract}
Stellar spectra have been obtained using a multichannel Fourier Transform Spectrometer (FTS) which incorporates components of the Navy Prototype Optical Interferometer. It is well known that a FTS can provide superior wavelength stability as compared to traditional spectrometers. Unfortunately the FTS traditionally suffers from exceptionally poor sensitivity, which until now has limited its uses to sources with high fluxes and/or those with narrow band emission (e.g. the Sun, nebulae, and laboratory samples). We present stellar observations using a new FTS design which overcomes this sensitivity limitation by using a conventional multichannel spectrometer in conjunction with the FTS system. The signal-to-noise ratio of spectra from our test-bed observations are consistent with the theoretical prediction and show that for $N$ channels the sensitivity scales like $N$, while the signal-to-noise ratio scales like $\sqrt{N}$. With this type of an instrument on a 3-m telescope and 9000 channels we expect to be able to detect and measure such exciting astrophysical phenomenon as gravitational redshifts from single, main sequence stars and extrasolar planets of terrestrial mass.
\end{abstract}

\section{Introduction}

A laser-metrology equipped Fourier Transform spectrometers (FTS) can provide greater frequency stability and spectral resolution than is practical with absorption-cell spectrometers. Unfortunately, a FTS does not possess the sensitivity of standard spectrometers. High spectral resolution observations of astronomical sources using FTS systems have been limited to very bright objects or narrow-band emitters. Relatively faint objects (NGC 4151; Rieke \& Lebofsky 1981) have been observed, but only at very low spectral resolution.

We retain the high spectral resolution available to a FTS while achieving the high sensitivity of normal spectrometers by coupling the latter to the former. We find that the sensitivity increases linearly with the number of spectral channels. By doing so, we expect to be able to detect the motion of Earth mass planets around only moderately bright stars and measure the masses of single stars from their gravitational redshift using the second order Doppler effect. These 


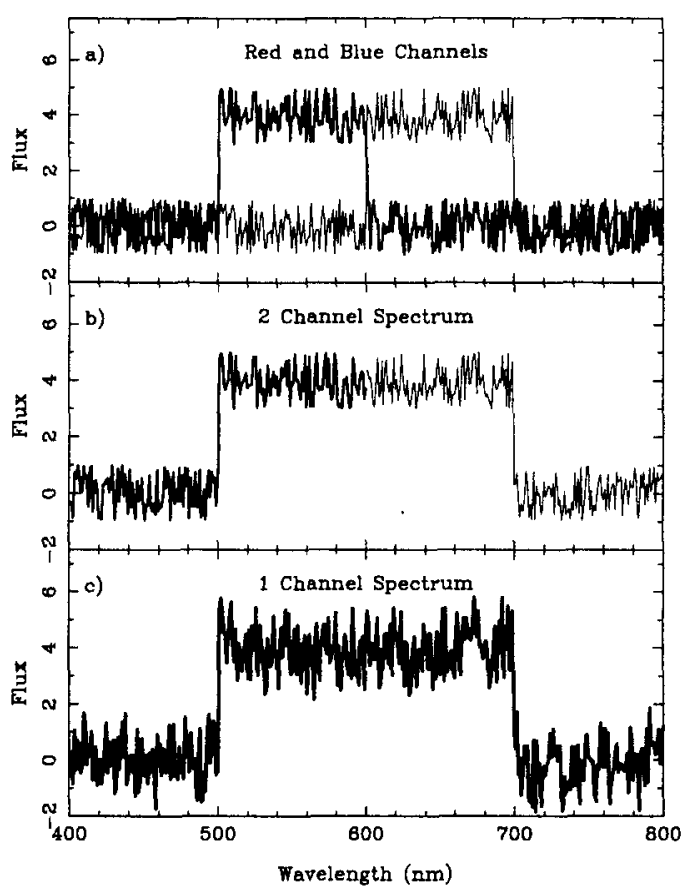

Figure 1. (a) Spectra from two channels with non-overlapping bandwidths. (b) A spectrum made by "pasting" together the signal from the two spectra in (a). (c) A single channel spectrum (i.e. the equivalent of a direct sum of the signal and noise from the spectra in (a)).

are objectives beyond the capability of even absorption-cell spectrometers on the largest telescopes.

We have utilized hardware and software in place at the Navy Prototype Optical Interferometer (NPOI) to convert it from a spatial interferometer into a temporal interferometer. We call this test-bed instrument the NPOI-mFTS. For this paper we define the following: (1) FTS: The traditional single channel Fourier Transform Spectrometer. (2) mFTS: A multichannel FTS (i.e. a FTS coupled with a multichannel spectrometer). (3) NPOI-mFTS: The mFTS built using elements of the NPOI.

\section{The Multichannel FTS and NPOI-mFTS}

In order to achieve high sensitivity while retaining high spectral resolution we have modified the FTS design. Precise velocity (wavelength) calibration is achieved by passing a high precision metrology laser through the optical delay lines to measure the optical path difference. The correlated beam from the FTS is then dispersed onto an array of detectors using a conventional spectrometer. Figure 1 shows graphically how the noise level in a 2-channel (and by extension, $N$-channel) FTS is less than that of the single channel FTS. 
Light is collected by a single 50 -cm flat mirror which is one of several comprising the NPOI (only $12 \mathrm{~cm}$ of aperture is currently illuminated). The light beam is split and sent down separate optical delay lines which consist of evacuated pipes which contain retro-reflectors mounted on movable carts. A metrology laser is used for precisely determining the cart position while one delay line is held fixed and the other is slewed at a constant rate. The two beams emerging from the delay lines are injected into the beam combiner. The beam combiner outputs yield direct measures of the photometry of the delay line signal plus the correlated output containing the interferogram. An array of lenslets redirects the outputs from the beam combiner onto a series of 32 detectors, which span $\lambda \lambda 850 \mathrm{~nm}$ to $450 \mathrm{~nm}$. Each detector is sampled at $4 \mathrm{kHz}$ while the delay lines are slewed.

\section{Observations and Signal to Noise}

We acquired broadband interferograms from Betelgeuse and Procyon with the NPOI-mFTS. Transformation of the interferograms using a FFT produced spectra of both Betelgeuse (Figure 2) and Procyon. The correspondence of the mFTS with a reference spectrum (Kiehling 1987) is excellent. Using a 12-cm diameter aperture $(d)$, and a 32-channel spectrometer $\left(R_{\mathrm{c}}\right)$ we achieved a signal-to-noise ratio in the spectrum $\left((S N R)_{\mathrm{S}}\right)$ of 210 with an integration time $\left(T_{\mathrm{int}}\right)$ of $1 \mathrm{hr}$ at a resolution $\left(R_{\mathrm{FTS}}\right)$ of $1500(200 \mathrm{~km} / \mathrm{s})$. The throughput of the NPOI $(\alpha)$ is estimated to be only $2.6 \%$.

From our observations we extrapolate the performance of the NPOI-mFTS to systems with more spectral channels and a larger aperture:

$$
(S N R)_{\mathrm{S}}=(210)\left(\frac{R_{\mathrm{c}}}{32}\right)^{\frac{1}{2}}\left(\frac{T_{\mathrm{int}}}{3718 \mathrm{~s}}\right)^{\frac{1}{2}}\left(\frac{R_{\mathrm{FTS}}}{1470}\right)^{-1}\left(\frac{\alpha}{2.6 \%}\right)^{\frac{1}{2}}\left(\frac{d}{12 \mathrm{~cm}}\right) 10^{\frac{0.5-m_{V}}{5}} .
$$

From Butler et al. (1996), a spectrum with $R=62000$ obtained with $(S N R)_{\mathrm{S}}=$ 200 over a total bandpass of $\Delta \lambda=140 \mathrm{~nm}$ from a solar-type star has a Doppler velocity uncertainty due to photon errors alone $\sigma\left(v_{\text {photon }}\right)=1.2 \mathrm{~m} / \mathrm{s}$. We can scale the expression for $\sigma\left(v_{\text {photon }}\right)$ :

$$
\sigma\left(v_{\text {photon }}\right)=\left(1.2 \mathrm{~m} \mathrm{~s}^{-1}\right)\left(\frac{200}{(S N R)_{\mathrm{S}}}\right)\left(\frac{140 \mathrm{~nm}}{\Delta \lambda}\right)^{\frac{1}{2}} .
$$

For bright stars $\left(m_{V}<6\right)$ and long integrations $(t>10 \mathrm{~min})$ systematic errors are important. Currently the smallest systematic errors are claimed by absorption-cell spectrometers at 1-2 m/s level. Sources of systematic errors for the mFTS are: (1) The frequency drift of the laser causes errors in the delay position for calculation of lag. For the NPOI-mFTS this limit is $0.16 \mathrm{~m} / \mathrm{s}$. This can be reduced by a few orders of magnitude with a higher precision model laser. (2) Thermal fluctuations result in the optical elements of the mFTS drifting with respect to one another. The observed thermal characteristics of the NPOI-mFTS produces an error at the $0.13 \mathrm{~m} / \mathrm{s}$ level (Hajian et al. 1998). This can be reduced by injection of the metrology laser at the telescope, so as to run through the entire optical system. 


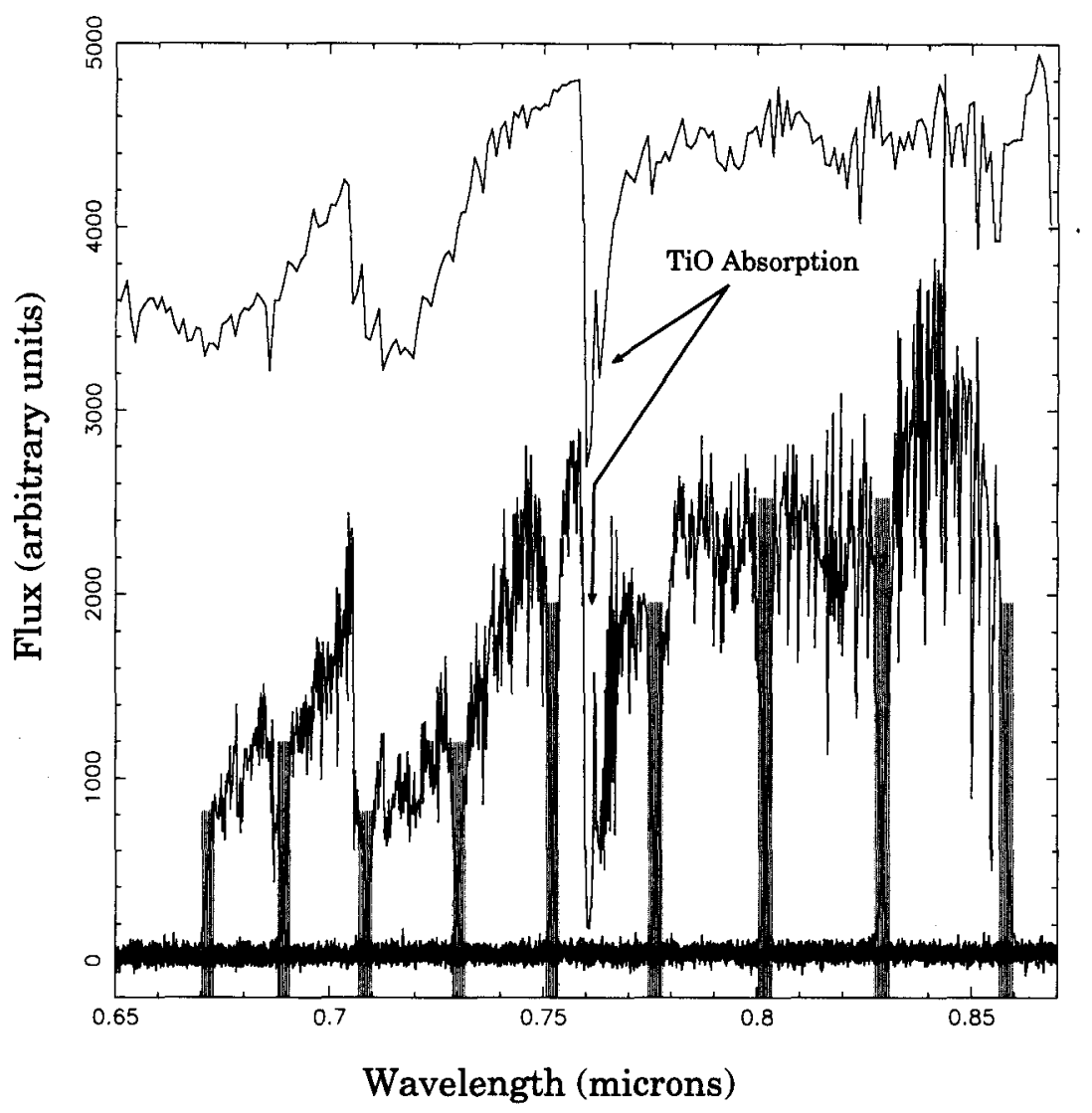

Figure 2. The mFTS spectrum from Betelgeuse using eight channels. Grey bands indicate gaps in the detector array. The upper spectrum is a reference spectrum from Kiehling (1987). 


\section{Objectives, Caveats and Conclusions}

A planet with approximately the mass and orbital radius of the Earth will induce a peak-to-peak reflex motion of $0.18 \mathrm{~m} / \mathrm{s}$ on its star. The goal of this project is to build an instrument with: $d=300 \mathrm{~cm}, R_{\mathrm{c}}=9000, \alpha=20 \%, R_{\text {FTS }}=62000$, and $\Delta \lambda=400 \mathrm{~nm}$.

For one hour of integration time on a 5.5 mag star we expect a $(S N R)_{\mathrm{S}}$ of 570 yielding a velocity uncertainty of $0.25 \mathrm{~m} / \mathrm{s}$. At this level of precision the detection of terrestrial mass planets by means of their reflex motion is within reach. At the very least we expect to determine the minimum detectable reflex motion due to an extrasolar planet (as a function of spectral type) while at the same time explore interesting stellar astrophysics.

\section{Discussion}

Dravins: The wavelength accuracy of an FTS will in practice not be limited by e.g. the laser stability, but by other factors. A multichannel system implies spectral dispersion at the optical input and/or output side. To restore or normalize a spectral portion requires knowledge of the sensitivity function for each channel. Many channels imply that these sensitivity funnctions will vary rapidly with wavelength, and be increasingly difficult to determine accurately. Other FTS effects include that the effective length of the interference cavity is slightly different for light at different wavelengths, possibly requiring lasers at multiple wavelengths for exact calibration.

Isaak: What is the maximum optical luminosity (étendue), in metre-arcseconds (say) of the instrument?

Hajian: Currently this has not been determined.

\section{References}

Armstrong, J.T., Mozurkewich, D., Rickard, L.J, Hutter, D.J., Benson, J.A., Bowers, P.F., Elias II, N.M., Hummel, C.A., Johnston, K.J., Buscher, D.F., Clark III, J.H., Ha, L., Ling, L.-C., White, N.M., \& Simon, R.S. 1998, AJ, 496, 550.

Butler, R.P., Marcy, G.W., Williams, E., McCarthy, C., Dosanjh, P., \& Vogt, S.S. 1996, PASP, 108, 500.

Hajian, A.R., Armstrong, J.A., Hummel, C.A., Benson, J.A., Mozurkewich, D., Pauls, T.A., Hutter, D.J., Elias II, N.M., Johnston, K.J., Rickard, L.J, \& White, N.M. 1998, ApJ, 496, 484

Kiehling, R. 1987, A\&AS, 69, 465.

Rieke, G.H. \& Lebofsky 1981, ApJ, 250, 87. 